\title{
Macroeconomic impacts of the public health response to COVID-19
}

Eric Kemp-Benedict

May 2020, revised May 2020 
Macroeconomic impacts of the public health response to COVID-19

Eric Kemp-Benedict, Stockholm Environment Institute

4 May 2020

\begin{abstract}
The economic impact of public health measures to contain the COVID-19 novel coronavirus is a matter of contentious debate. Given the high uncertainties, there is a need for combined epidemiologicalmacroeconomic scenarios. We present a model in this paper for developing such scenarios. The epidemiological sub-model is a discrete-time matrix implementation of an SEIR model. This approach avoids known problems with the more usual set of continuous-time differential equations. The postKeynesian macroeconomic sub-model is a stylized representation of the United States economy with three sectors: core, social (most impacted by social distancing), and hospital, which may experience excessive demand. Simulations with the model show the clear superiority of a rigorous testing and contact tracing regime in which infected individuals, symptomatic or not, are isolated. Social distancing leads to an abrupt and deep recession. With expanded unemployment benefits, the drop is shallower. When testing and contact tracing is introduced, social spending can be scaled back and the economy recovers quickly. Ending social distancing without a testing and tracing regime leads to a high death toll and severe economic impacts. Results suggest that social distancing and fiscal stimulus have had their desired effects of reducing the health and economic impacts of the disease.
\end{abstract}

Keywords: SARS-CoV-2; coronavirus; COVID-19; macroeconomy; post-Keyensian; SEIR model JEL codes: E00, E11, I18

Acknowledgements: I would like to thank Antoine Godin for critical comments on the macroeconomic model. His suggestions led to a substantial reworking of parts of the analysis. All remaining errors are my own. 


\section{Introduction}

Actions to slow the spread of the COVID-19 novel coronavirus are sharply reducing economic activity, thereby putting people out of work, placing significant strains on businesses, and threating a deep recession. In this rapidly changing situation there is a need for analytical tools to better understand the economic impacts of public health measures. This paper presents a version of such a tool: a combined epidemiological-macroeconomic simulation model. Given substantial ongoing uncertainty about the epidemic, the model is necessarily stylized. Nevertheless, for illustration and to anchor to a real case as best as possible, we calibrate to the United States and reference the US context when making modeling decisions.

The model proposed in this paper is post-Keynesian, sometimes styled "heterodox", in contrast to more "orthodox" neoclassical models. The neoclassical models that have been produced to date are optimizing models in which individuals make choices that maximize their intertemporal utility. Following convention, workers in these models are assumed to derive utility from consumption and disutility from work. In most such models, the primary impact of the disease is on the supply side: workers do not go to work. In the models of Morin et al. (2018) and Eichenbaum et al. (2020), individuals choose between engaging in economic activities (which expose them to the risk of infection) and distancing themselves. Eichenbaum et al. also consider demand effects, as people reduce consumption to avoid exposure, building in an estimation of utility while infected or recovered. Morin et al. argued that through a combination of positive externalities - reducing others' exposure to the disease - and negative externalities - reducing economic activity personal choices are socially sub-optimal. The role of policy is to alter the personal costs and benefits to shift behavior in socially optimal ways.

The pre-pandemic model of Morin et al. and the early pandemic model of Eichenbaum modeled the effect of policies through a tax, which serves as a proxy for the internal calculations of individuals as they respond to public health measures (Eichenbaum et al. 2020, p.5). For example, Morin et al. (2018, p.287) suggest mandatory sick leave without pay as a policy to increase personal avoidance measures. This approach becomes difficult with directive policies such as shelter-in-place ordinances, because such models would require modified individual utility functions to include a trade-off between the benefits of flouting the law and the costs of the penalty. Rather than go this route, more recent models, such as that of Fornaro and Wolf (2020) and Guerrieri et al. (2020) directly include the possibility that people may be forbidden to go to work.

A question raised in the papers of Fornaro and Wolf and Guerrieri et al. is the extent to which the supply shock - that is, a change in the number of workers showing up for work - translates into a demand shock a change in demand for goods and services. The optimizing framework these models employ makes such a linkage unlikely, because prices are assumed to adjust to bring the economy to full utilization. The authors therefore invoke additional mechanisms that block full adjustment. Fornaro and Wolf (2020) follow recent debates on "secular stagnation" (Summers 2014; Teulings and Baldwin 2014) and propose a limit to price flexibility: the zero lower bound to the interest rate. Guerrieri et al. (2020) emphasize the fact that employment cannot respond flexibly in a sector that has been shut down and the goods it produces cannot be bought at any price.

Constraining the price-driven adjustment mechanisms allow optimizing models to produce outcomes qualitatively similar to those we observe in the real economy, but also illustrate the lengths to which the optimizing behavior must be modified in order to mimic real-world outcomes. This exemplifies well-known limitations of neoclassical macroeconomic models (Shaikh 2016, chap.3; Solow 2008) with their 
underlying rational actor assumption (Kirman 2014), made in the service of (problematic) "microfoundations" (King 2012). We do acknowledge that individuals appear to make a personal assessment of risk when deciding on ordinary health protective measures, such as vaccinations and checkups (Janz and Becker 1984). Such documented individual decision-making can inform a worker choice model, as argued by Morin et al. (2018, p.286). However, given the rather clear and extraordinary motivations for avoiding gathering in dense groups, we find it more plausible with the COVID-19 pandemic to directly represent the impact of public health measures without passing through a questionable individual optimizing calculation as an intermediate step.

In the post-Keynesian model presented in this paper, we represent public health measures as causing a fall in demand. Certainly both supply and demand factors are active. The spread of the disease throughout the world is disrupting global supply chains, while maintaining a safe distance, even in businesses deemed "essential", means lower productivity. ${ }^{1}$ Moreover, simulations with the model developed in this paper exhibit supply-chain constraints on hospitals if COVID-19 is allowed to spread unchecked.

Nevertheless, from the point of view of a business deemed "non-essential", requiring the business to close its consumer-facing outlets is more similar to a withdrawal of demand for its goods or services than it is a withdrawal of willing labor. Even without an order, public health messages encourage people to stay home as much as possible, which is directly experienced as a loss of demand. Indeed, we can question whether the primary impact of the public health response is a loss of labor supply due to an individual utilitymaximizing calculation. At least in the US, when faced with rental payments and expenditure on food, utilities, and other necessities, many people have no option of withdrawing their labor. The cost of necessities is not small. For the US population as a whole, the total of shelter, food at home, utilities, health insurance, and medications accounted for $39 \%$ of expenditure in $2018 .{ }^{2}$ For the lowest-earning fifth of the surveyed population, those categories accounted for half of expenditure. Under a shelter-at-home order, households will likely see that share rise as they spend more on food at home and utilities, while they cannot easily reduce spending on rent, health insurance, or medication. Without sufficient income, they must curtail other expenditure, even on such necessities as clothing. If they cannot negotiate a reduction or deferral of rent payments, they may face eviction.

The loss of demand in directly-impacted businesses leads to a loss of business income, layoffs and furloughs. In post-Keynesian theory, firms are assumed to plan their investment in anticipation of demand, making such models "demand-led". This suggests that directly impacted sectors will curtail investment, itself a source of demand. As firms in those sectors lay off workers, the fall in wage income means lower spending on the goods and services offered by firms that are not directly impacted. Moreover, to the extent that investment is curtailed, the firms who would have produced the investment goods will see their revenues fall, potentially leading to further job losses and a further decline in investment. That is not true of all firms - some sectors may benefit from the increase in online activities and purchases. Nevertheless, a loss of demand has a potentially substantial multiplier effect both through consumption and investment expenditure.

For households that could purchase goods and services but do not because of public health measures, the counterpart to reduced expenditure is greater saving. That saving can fund an expansion of unemployment

\footnotetext{
${ }^{1}$ Some food processing plants have not altered their production routines and have been hotspots for COVID-19 (https://www.washingtonpost.com/business/2020/04/28/trump-meat-plants-dpa/, accessed 30 April 2018).

${ }^{2}$ U.S. Bureau of Labor Statistics Table 1110. Deciles of income before taxes: Annual expenditure means, shares, standard errors, and coefficients of variation, Consumer Expenditure Survey, 2018.
} 
benefits and other stimulus, keeping the money in circulation, supporting households who have lost income, and buffering the decline in GDP.

The model presented in this paper is a simulation model, rather than an optimizing model. It seeks to simulate the macroeconomic impacts of the epidemic and of the public health measures to contain it. Reductions in demand due to infection and sheltering in place drive a deep recession that is enhanced through multiplier effects. Aggressive social distancing significantly reduces the number of deaths but leads to a stronger recession. A fiscal stimulus helps households maintain needed expenditure until an effective testing and contact tracing regime is in place.

\section{Epidemiological model}

We use an aggregate or "compartmental" epidemiological model in this paper. Such models track different fractions of a population who are at different stages with respect to the disease. The earliest and simplest such model (Kermack and McKendrick 1927) includes susceptible, infective, and recovered (SIR) populations. COVID-19 is characterized by a long incubation period, so a modification to SIR, the SEIR model, is used most often (e.g., Fang et al. 2020; Li and Feng 2020; Rocklöv et al. 2020; Zhang et al. 2020). The SEIR model adds an "exposed" population to the other three that has contracted the virus but is both asymptomatic and non-infectious. Analyses of COVID-19 have extended the SEIR model to, e.g.: add a "confirmed" category (Zhan et al. 2020); implement exposed population dynamics using a time delay (Menendez 2020); explicitly model quarantine (Chatterjee et al. 2020); track populations by age groups (Matrajt and Leung 2020); and consider a variety of partially and fully infectious pools (Chowell et al. 2020). Alternative modeling strategies include a regression model (Gupta et al. 2020), a Bayesian model that takes observed country experiences as priors (Liu and Guo 2020), and, importantly for the US context, a statistical cumulative death rate model (the IHME model: IHME COVID-19 health service utilization forecasting Team and Murray 2020). The IHME model, which is continually updated, is reportedly an important input into planning by the US Federal Administration.

Compartmental models are normally represented by a system of continuous-time coupled nonlinear differential equations. However, this approach has well-documented problems (Lloyd 2001a; Lloyd 2001b; Wearing et al. 2005). Of particular concern is that people move out of the infective pool with an exponential decay. There is therefore never a time when the infective population goes to zero, even if the introduction of new cases through in-migration is brought to zero. Grant (2020) proposes an alternative, matrix, formulation in which the time in days since exposure and since becoming infective is tracked explicitly. We follow Grant by constructing a discrete-time matrix SEIR model of epidemiological dynamics.

We make a further change that is particularly important in a large country such as the US: uneven spread of the disease. The SEIR model assumes that any susceptible individual has the same probability of encountering an infected individual as any other susceptible individual. This implies that the infected population is evenly distributed throughout the population. In reality, the infected population is unevenly distributed, so some epidemiological models, such as the IHME model, use state-level data. We modify the standard SEIR model to take inhomogeneity into account at a national aggregate level.

We offer an important caveat: Only epidemiological models accepted by epidemiologists should be used as a basis for forecasts of cases and deaths during the pandemic. ${ }^{3}$ Models used for policy are continually updated and spatially disaggregated. We have attempted to make good use of existing models and

\footnotetext{
${ }^{3}$ The US Centers for Disease Control (CDC) provide a continually updated set of outputs from a suite of models https://www.cdc.gov/coronavirus/2019-ncov/covid-data/forecasting-us.html.
} 
epidemiological studies in constructing the epidemiological sub-model, but the focus of this paper is economics.

\subsection{Matrix SEIR model}

Both the epidemiological and macroeconomic models run at a daily time step. The SEIR model splits the total population $N$, which declines due to mortality from the disease, into a susceptible population $N_{s}$, an exposed population $N_{e}$, an infective population $N_{i}$, and a recovered (and presumed immune) population $N_{r}$. We ignore normal mortality, but estimate excess mortality due to the disease. We also ignore births and immigration.

Following Grant (2020, p.4), people move from the susceptible to the exposed category at a modeldependent rate that we denote $r_{e}$. There is a maximum number of days for incubation, $m$, and a maximum number of days for remaining infective, $n .{ }^{4}$ It is possible for individuals to move out of the exposed category or the infective category earlier than that, as given by fractions of the population transitioning from one category to the next. We indicate these fractions below with the symbols $r_{i t}$ and $r_{r t}$. Infective individuals may also die from the disease, at a daily rate $d_{t}$. In contrast to Grant, we do not allow for reinfection or loss of immunity. Our matrix model is given by the following equation, where a "+1" subscript means a subsequent day and parenthesized numbers in superscripts is the days since exposure or infection.

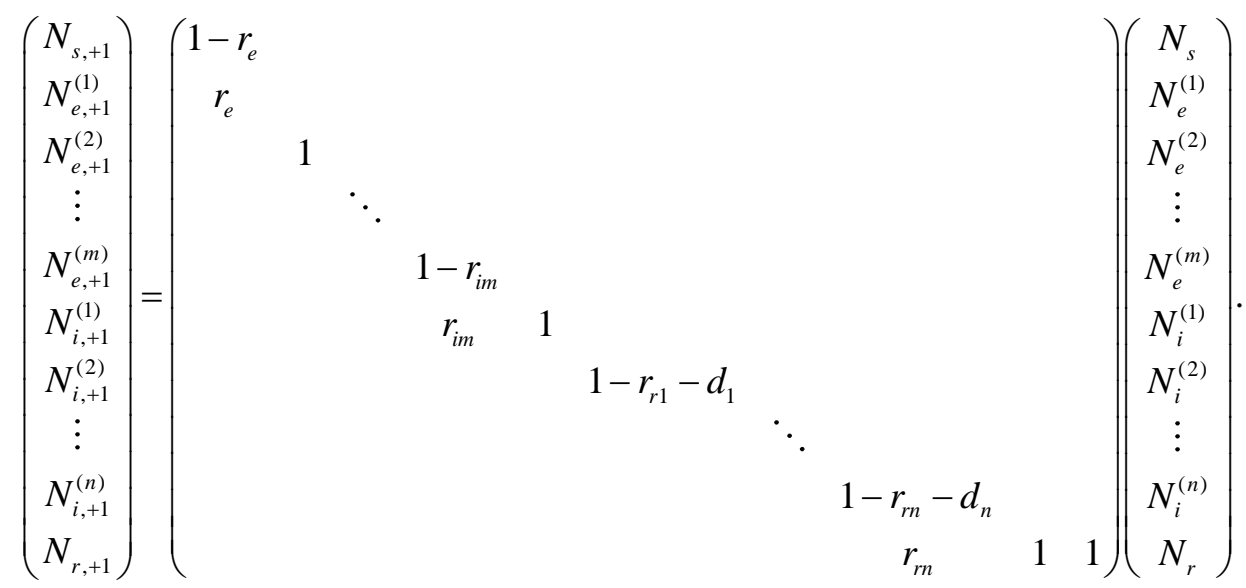

The incubation period of COVID-19 has been estimated to have a median of 5.1 days, with $95 \%$ of cases exhibiting symptoms between 2.2 and 11.5 days after exposure (Lauer et al. 2020). We approximated Lauer et al.'s curve by setting $r_{i t}$ to different values when passing from day 1 to day 2 , day 2 to day 3 , and so on. The recovery period (or time to death) covers a wider range. Wang et al. (2020) documented a comparatively small number (17) of fatal cases and found a range of 6 to 41 days from the onset of symptoms to death, with a median of 14 days and a mean of $T_{i}=16$ days. Of those, $90 \%$ of cases lasted between 7 and 32 days. We set the total of $r_{r t}$ and $d_{t}$ to different values to mimic that curve. We assume that patients either recover or die along that curve in the same proportion. Thus, we allocate between recovery and mortality with a fixed ratio $d$, such that

$$
d_{t}=d\left(r_{r t}+d_{t}\right), \quad r_{r t}=(1-d)\left(r_{r t}+d_{t}\right)
$$

${ }^{4}$ For Grant, $m=2, n=4, r_{e}$ is denoted by $X, r_{i}=0.100$, and $r_{r}=0.003+0.277=0.300$. 


\subsection{Exposure rate}

In the SEIR model, the exposure rate $r_{e}$ depends on the size of the susceptible and infected populations. The critical parameter is the probability of exposure per encounter between a susceptible and infected individual, $\beta$, which depends on behavior as well as the characteristics of the disease. We separate this into two factors, a baseline rate $\beta_{0}$, in the absence of any public health interventions, and an adjustment factor that captures the effect of interventions.

A key uncertainty in the progress of the COVID-19 pandemic is how many people have already been infected. There are indications that the number may be quite large, perhaps exceeding confirmed cases by a factor of $10 .{ }^{5} \mathrm{We}$ therefore introduce as a model parameter the fraction $f_{v}$ of "visible" cases. This parameter does a lot of work in the model. It is conceptually closest to the fraction of confirmed cases, but we also use it as a proxy for symptomatic cases. We expect these to be largely overlapping categories, with the set of symptomatic cases larger than the set of confirmed cases. We calibrate it to match cumulative deaths from COVID-19 in the US and find a value of $f_{v}=13 \%$.

We set the value of $\beta_{0}$ based on an assumed basic reproductive number $R_{0}$ and the mean infectious period $T_{i}$ as

$$
\beta_{0}=\frac{R_{0}}{T_{i}}
$$

We set $R_{0}=2.25$, half-way between the lower and upper limits in Moghadas et al. (2020). Using the mean $T_{i}$ from Wang et al. (2020) gives $\beta_{0}=0.14 /$ day. The adjustment factor depends on the particular public health measures adopted. We consider three: social distancing, or self-isolation, to an extent $\sigma_{\mathrm{SD}}$; the isolation of visible individuals, given by $f_{v}$; and (through testing and contact tracing) the isolation of a fraction $\sigma_{\mathrm{IC}}$ of infectious cases. When all three are in place, the factor $\beta$ is given by

$$
\beta=\left(1-\sigma_{\mathrm{SD}}\right)\left[1-\max \left(f_{v}, \sigma_{\mathrm{IC}}\right)\right],
$$

This expression captures the reduction in the number of interactions a susceptible person will have (1 $\left.\sigma_{\mathrm{SD}}\right)$ and the fraction of infective individuals they might encounter $\left(1-f_{v}\right.$ or $\left.1-\sigma_{\mathrm{IC}}\right)$.

\subsection{Inhomogeneity}

To represent uneven distribution of the disease, we treat the total population as separated into a large number of localities. For the $j$ th locality, we denote the total population by $n_{j}$, the susceptible population by $n_{j s}$, and so on. Within that locality, the rate of exposure per member of the susceptible population, $r_{e j}$, is given by the product of the probability of encountering an infective individual per encounter - assumed in the SEIR model to be the ratio of the infected to the total population - and the probability of becoming exposed given that encounter, which is the parameter $\beta$,

$$
r_{e j}=\beta \frac{n_{j i}}{n_{j}}
$$

Applying the matrix equation (1), the susceptible population in the subsequent day is

\footnotetext{
${ }^{5}$ See, e.g.: https://www.nejm.org/doi/full/10.1056/NEJMe2009758 (accessed 30 April 2020) and https://www.washingtonpost.com/health/antibody-tests-support-whats-been-obvious-covid-19-is-much-more-lethalthan-flu/2020/04/28/2fc215d8-87f7-11ea-ac8a-fe9b8088e101_story.html (accessed 30 April 2020).
} 


$$
n_{j s,+1}=\left(1-r_{e j}\right) n_{j s}=n_{j s}-\beta \frac{n_{j s} n_{j i}}{n_{j}}
$$

For the second, nonlinear, term, we note that the sum of susceptible, exposed, infected, and recovered must equal the total population, so we can replace $n_{j s}$ with

$$
n_{j s}=n_{j}-n_{j e}-n_{j r}-n_{j i}
$$

Substituting into equation (5), and summing over all localities, we have

$$
N_{s,+1}=\sum_{j} n_{j s,+1}=N_{s}-\beta \sum_{j} n_{j i}+\beta \sum_{j} \frac{\left(n_{j e}+n_{j r}\right) n_{j i}}{n_{j}}+\beta \sum_{j} \frac{n_{j i}^{2}}{n_{j}} .
$$

The first sum on the right-hand side is simply $N_{i}$. For the second, we make the simplifying assumption that the distribution of exposed and recovered are uncorrelated with the distribution of infected across localities. The average of their products can then be replaced with the product of the averages, in which case the sum becomes $\left(N_{e}+N_{r}\right) N_{i} / N$. Adding and subtracting a term $N_{i}^{2} / N$, we have

$$
\begin{aligned}
N_{s,+1} & =N_{s}-\beta \frac{\left(N-N_{e}-N_{r}-N_{i}\right) N_{i}}{N}+\beta \sum_{j} \frac{n_{j i}^{2}}{n_{j}}-\beta \frac{N_{i}^{2}}{N} \\
& =N_{s}-\beta \frac{N_{s} N_{i}}{N}+\beta \sum_{j} \frac{n_{j i}^{2}}{n_{j}}-\beta \frac{N_{i}^{2}}{N} .
\end{aligned}
$$

The difference from equation (6) at the national level is in the last two terms. To simplify them, note that

$$
\sum_{j} n_{j}\left(\frac{n_{j i}}{n_{j}}-\frac{N_{i}}{N}\right)^{2}=\sum_{j} \frac{n_{j i}^{2}}{n_{j}}-2 \frac{N_{i}}{N} \sum_{j} n_{j i}+\frac{N_{i}^{2}}{N}=\sum_{j} \frac{n_{j i}^{2}}{n_{j}}-\frac{N_{i}^{2}}{N} .
$$

The final expression is the last two terms in equation (9), aside from the factor $\beta_{0} k$. Meanwhile, the expression on the left hand side can also be written

$$
\sum_{j} n_{j}\left(\frac{n_{j i}}{n_{j}}-\frac{N_{i}}{N}\right)^{2}=\frac{N_{i}^{2}}{N} \sum_{j} \frac{n_{j}}{N}\left(\frac{n_{j i}}{n_{j}} \frac{N}{N_{i}}-1\right)^{2}=\frac{N_{i}^{2}}{N} c_{i}^{2},
$$

where $c_{i}$ is the coefficient of variation of the infected share of the population across localities. Putting this together, we have

$$
N_{s,+1}=N_{s}-\beta \frac{N_{s} N_{i}}{N}+\beta \frac{N_{i}^{2}}{N} c_{i}^{2}=N_{s}\left[1-\beta \frac{N_{i}}{N}\left(1-\frac{N_{i}}{N_{s}} c_{i}^{2}\right)\right] .
$$

If there is no variation in the infective population between sites, then $c_{i}=0$ and this reduces to the expression for an individual location in equation (6). Otherwise the inhomogeneity of the infective population tends to lower the national average exposure rate. The result is a modified expression for the national exposure rate,

$$
r_{e}=\beta \frac{N_{i}}{N}\left(1-\frac{N_{i}}{N_{s}} c_{i}^{2}\right)
$$




\subsection{Mortality rate}

We represent the mortality rate $d$ as a product of three factors: the fraction of visible cases $f_{v}$, the hospitalization rate $\eta$ per visible case, and the mortality rate per hospitalized patient $\mu$. For the hospitalization rate per visible case we calculated a demographically-weighted average rate of mildly symptomatic cases using age-specific rates from (Moghadas et al. 2020 Table A5). One less than that comes to $\eta=38 \%$.

We further allow for the possibility that the mortality rate rises when hospitals overflow and beds become unavailable (Moghadas et al. 2020). When beds are available, hospitalized patients experience a baseline mortality rate $\mu_{0}$. However, for those who cannot be provided a bed due to overflow, the mortality rate rises to a higher level $\mu_{b}$. This appears to have been the case in Hubei province in China (Ji et al. 2020), which experienced an average mortality rate of $2.9 \%$ as against other provinces, where the average was $0.7 \%$.

Baud et al. (2020) estimated confirmed case fatality rates within and outside China. They found that case fatality rates have been exceedingly high, around $15 \%$, outside China, but that they were converging over time to a value close to $5.7 \%$, again suggesting higher mortality when health care systems are under stress. We calculate the baseline mortality rate per hospitalized patient as

$$
\mu_{0}=\frac{5.7 \%}{\eta} \text {. }
$$

We set the bed overflow rate as a multiple of the baseline. The findings reported by Ji et al. (2020) and Baud et al. (2020) suggest a factor of three, but practices have improved since the first cases. We assume a factor of two, setting $\mu_{b}=2 \mu_{0}$. Interpreting the fraction of visible cases as a proxy for confirmed cases and using the value of $f_{v}=13 \%$ reported above gives a baseline mortality rate of $0.74 \%$ per infected individual. That rate rises to $1.48 \%$ when hospitals are severely crowded.

In any particular locality, there will be a normal level of hospital bed occupancy from causes other than the pandemic. As some of those cases are postponed - for example, for elective surgery - the stock of available beds will expand. To simplify the model we assume the total number of hospital beds per person to be the same value, $b$, in each locality. Of those, there is normally an excess $\varepsilon$, which rises during the pandemic to a higher level $\varepsilon^{\prime}$. The number of available beds per person is then equal to $\varepsilon^{\prime} b$. There is overflow if the number of hospitalized infected patients exceeds that value. For locality $j$, that happens when

$$
\eta \frac{n_{j i}}{n_{j}}>\varepsilon^{\prime} b
$$

This gives a threshold value for the infected fraction $f_{j i}=n_{j i} / n_{j}$ of

$$
f_{i}^{\text {thresh }}=\frac{\varepsilon^{\prime} b}{\eta}
$$

Above, we introduced the coefficient of variation of the infected fraction across localities. We now introduce an explicit probability distribution. The variable of interest - the infected fraction - is bounded. However, we use a normal distribution and require the coefficient of variation to be less than 0.39. (In model runs, we assume a value of 0.30.) That ensures that the minimum of the $99 \%$ confidence interval is positive. With this assumption, the probability in locality $j$ that the infected fraction will exceed the threshold is 


$$
P\left(f_{j i}>f_{i}^{\mathrm{thresh}}\right)=1-\Phi\left[\frac{1}{c_{i}}\left(\frac{f_{i}^{\mathrm{thresh}}}{\bar{f}_{i}}-1\right)\right], \quad \bar{f}_{i}=\frac{N_{i}}{N}
$$

where $\Phi$ is the cumulative normal distribution function. The mean exceedance of the threshold per infected person, $E_{i}$, is given by

$$
E_{i}=\frac{1}{\bar{f}_{i}} E\left(f_{i}-f_{i}^{\text {thresh }} \mid f_{i}>f_{i}^{\text {thresh }}\right)=\int_{f_{i}^{\text {thresh }}}^{\infty} d f \frac{1}{\sqrt{2 \pi} c_{i}}\left(\frac{f_{i}}{\bar{f}_{i}}-\frac{f_{i}^{\text {thresh }}}{\bar{f}_{i}}\right) \exp \left[-\frac{1}{2 c_{i}^{2}}\left(\frac{f_{i}}{\bar{f}_{i}}-1\right)^{2}\right] .
$$

It is possible to show ${ }^{6}$ that this expression can be written in terms of the $z$-value as

$$
E_{i}=-c_{i} z_{i}\left(1-\Phi\left(z_{i}\right)\right)+\frac{c_{i}}{\sqrt{2 \pi}} e^{-\frac{1}{2} z_{i}^{2}}, \quad z_{i} \equiv \frac{1}{c_{i}}\left(\frac{f_{i}^{\text {thresh }}}{\bar{f}}-1\right) .
$$

The average mortality rate at the national level is then

$$
d=\eta\left[\left(1-E_{i}\right) \mu_{0}+E_{i} \mu_{b}\right]
$$

\subsection{Epidemiological scenarios}

We ran the model using the parameters outlined above for an epidemiological baseline scenario and three variants: isolating visible cases (Epi IV), implementing a 90\% effective testing and contact tracing regime to isolate infectious individuals (Epi II90), and social distancing that reduces contacts by three-quarters (Epi SD75). In all scenarios, interventions begin on March 28 and ramp up to full implementation after one month. Scenario estimates of cumulative deaths are shown in Figure 1, together with data and estimates from two models - IHME-CurveFit and LANL-GrowthRate - as reported by the CDC. ${ }^{7}$ The Epi SD75 scenario fits the CDC's Covid hub ensemble best. The very high estimated deaths in the Epidemiological baseline and Epi IV scenarios show the benefits of social distancing. Without the efforts taken to "flatten the curve", the number of deaths would be much higher.

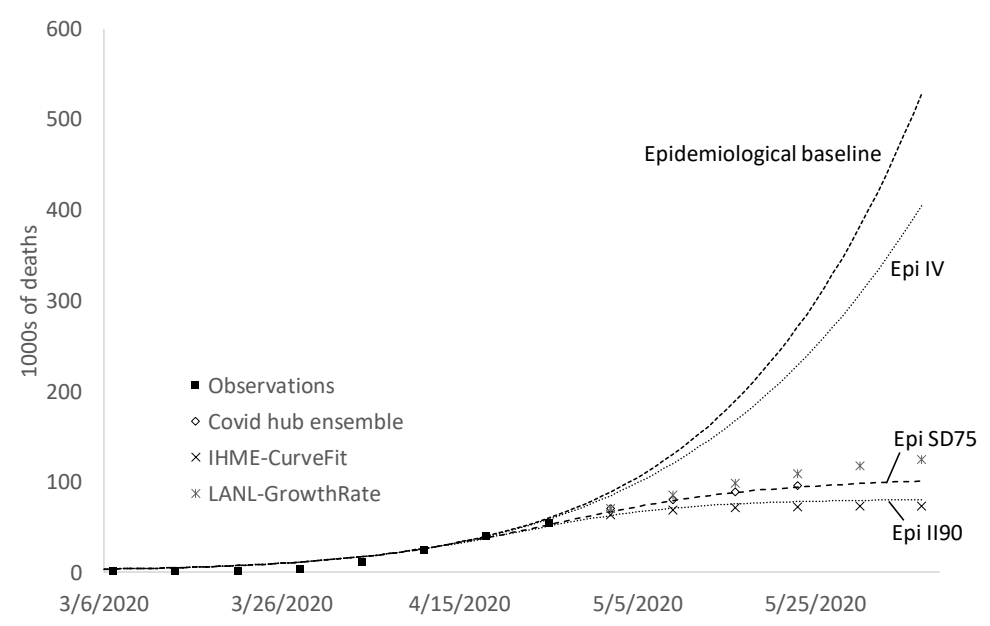

Figure 1: Cumulative deaths in model runs, compared with observed and model estimates as reported by the CDC

${ }^{6}$ The result can be found by taking the derivative of the expectation of $e^{a p}$ with respect to $a$ and setting $a$ to zero.

${ }^{7}$ https://www.cdc.gov/coronavirus/2019-ncov/covid-data/forecasting-us.html, accessed 3 May 2020. 
The number of deaths per day is shown in Figure 2. Under the Epi SD75 scenario, peak deaths per day has already passed. However, that assumes social distancing measures continue indefinitely. If they do, then cumulative deaths reach 109 thousand in the model simulation. If they are lifted without an effective testing and contact tracing regime in place, then the large remaining susceptible population will begin to encounter the small infective population and, as shown below, cases and deaths rise rapidly.

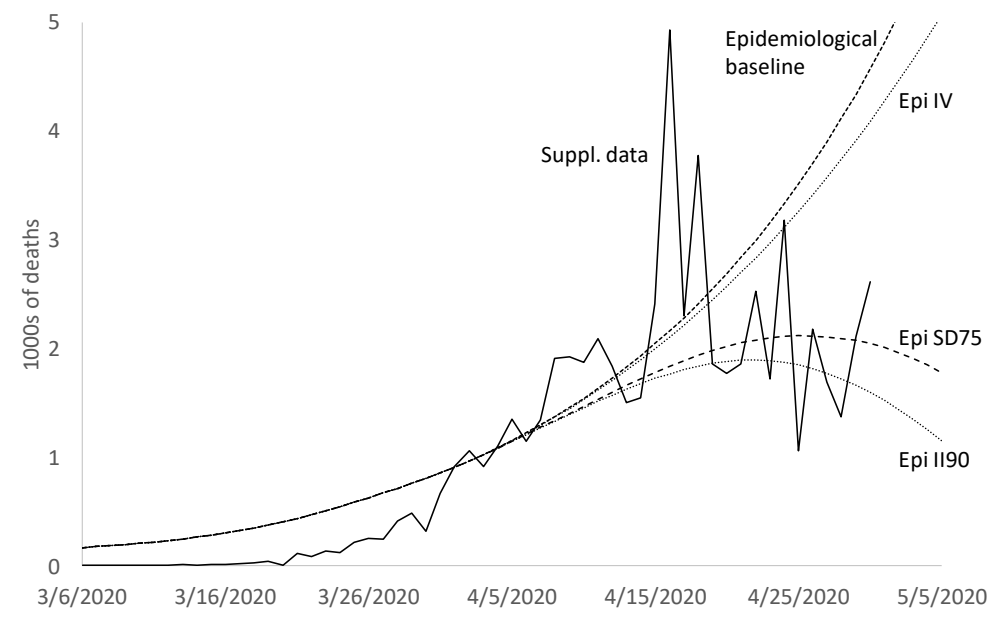

Figure 2: Deaths per day as simulated in the model compared with observations

The number of new visible cases in the model runs is shown in Figure 3. Also shown in the figure is the number of new cases as calculated from cumulative cases reported by the Centers for Disease Control (CDC). ${ }^{8}$ As shown in the figure, the number of reported cases starts more slowly than the model curve and then accelerates past it. That likely reflects the approximate way in which we treated heterogeneity; in the early stages of the disease the distribution was highly unevenly distributed, centered mainly in New York. The discrepancy may also arise in part from incomplete reporting early in the epidemic, while better and more extensive testing and reporting in recent weeks may explain the steady observed rate compared to the declining Epi SD75 scenario.

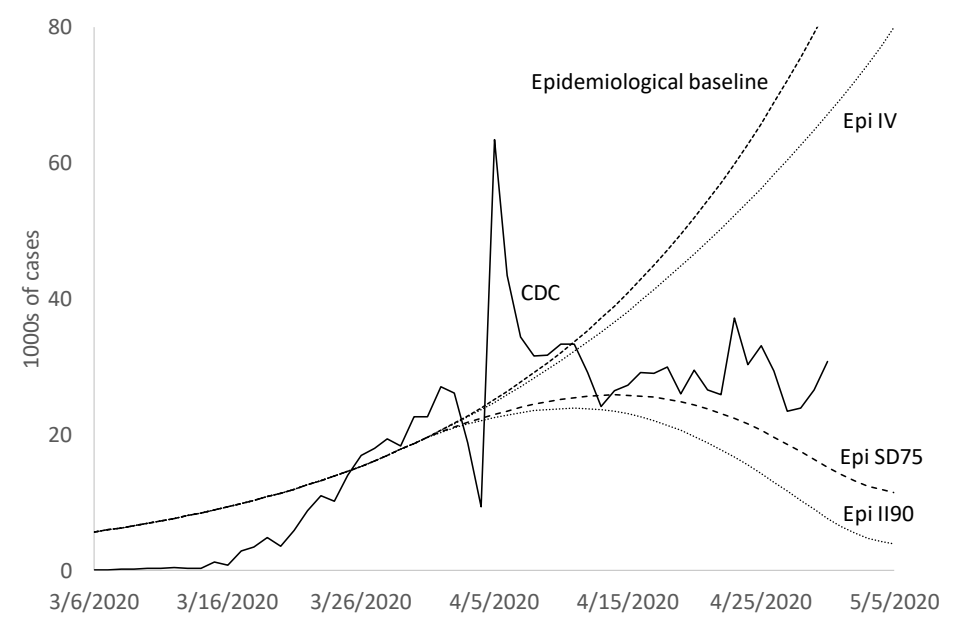

Figure 3: New visible cases in the scenarios, compared with the confirmed cases as reported by the CDC

${ }^{8}$ https://www.cdc.gov/coronavirus/2019-ncov/cases-updates/cases-in-us.html, accessed 23 April and 2 May 2020. 
As illustrated in Figure 1, isolating infective individuals is very effective at controlling the disease. However, that requires an effective testing and contact tracing regime, as called for in the US guidelines for reopening. ${ }^{9}$ If social distancing measures are removed without testing and tracing, the number of deaths will resurge. Simulations from the model are shown in Figure 4 for an "Epi SD75 + ending social distancing" scenario in which social distancing ends on June 15 with no testing and tracing regime. The simulation results are a sobering reminder that the size of the "second wave" will depend on how effectively the susceptible population can remain sequestered from the infective population.

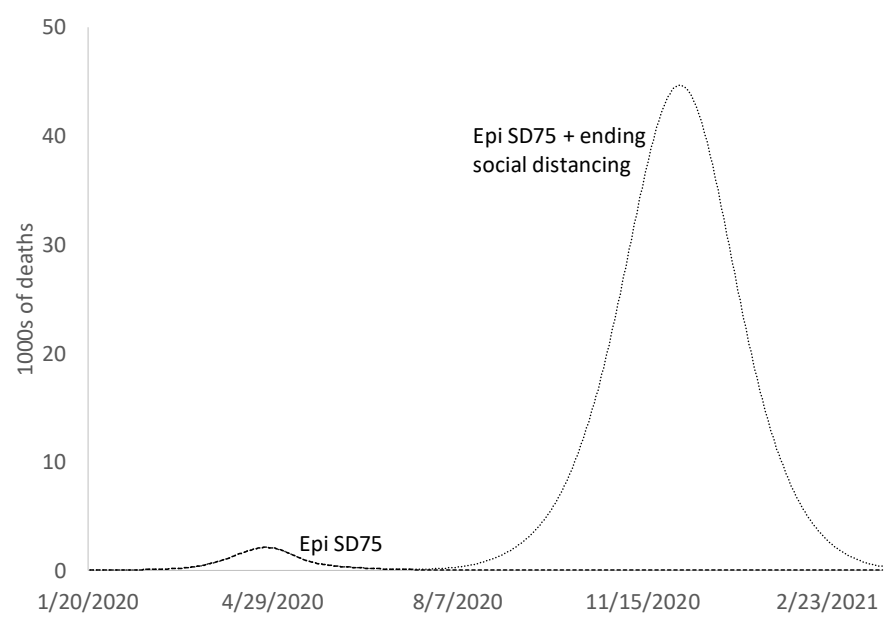

Figure 4: The effect of relaxing social distancing without testing and contact tracing

\section{Macroeconomic model}

The model economy has three sectors: core, social, and hospital. The "core" of the economy contains most economic activity. It provides the bulk of household goods and services, intermediate products used in all of the sectors (including the core sector itself), and investment goods. The "social" sector is most affected by sheltering in place. Furthermore, we assume that while people will spend down savings or go into debt to pay for core expenditures, they will not do so for social expenditures, so the social sector is most likely to be cut back when households have to curb their expenditure. The "hospital" sector has a baseline level of activity and it becomes more active as COVID-19 cases rise. Throughout, we compare to a baseline in which all components of the economy, including the wage bill, the capital stock, government expenditure, and sector output grow at (or very close to) an exogenous rate of $\gamma=2.0 \% /$ year, close to the recent average growth rate in real US GDP.

We attempt to capture an important issue in the US experience of the pandemic. As hospitals lose their usual patients, it frees up beds but reduces revenue, as many COVID patients cannot pay. As a result, costs are high, while profits are low or negative. We reflect this in incomplete cost recovery for the hospital sector in the model detailed below.

\subsection{Production and income}

The accounting framework for the model is conventional. Total net output (GDP) is the sum of value added in the core, social, and hospital sectors. Because this model has intermediate products, the sum of total output from each sector exceeds GDP. We denote the three sectors with subscripts: core (c), social (s), and hospital (h). We work in real quantities, assuming no change in relative prices between sectors.

${ }^{9}$ https://www.whitehouse.gov/openingamerica/, accessed 24 April 2020. 
The core sector produces goods for its own intermediate use, as intermediate inputs to the other two sectors, for some personal consumption, government purchases, and investment. Part of personal consumption and investment is provided by imports, at a net rate $m$. A part of personal consumption expenditure is paid as a sales tax. Drawing on input-output data for the US, ${ }^{10}$ we estimate a rate of $\tau_{c}^{\text {sales }}=5.6 \%$. With these assumptions, output $X_{c}$ is given by

$$
X_{c}=a_{c} X_{c}+a_{s} X_{s}+a_{h} X_{h}+G_{c}+(1-m)\left[\left(1-\tau_{c}^{\text {sales }}\right) C_{c}+I\right]
$$

In this expression $X_{s}$ and $X_{h}$ are output from the social and hospital sectors, $G_{c}$ is government expenditure on core goods, $C_{c}$ is personal expenditure on core goods, and $I$ is total investment expenditure. Using inputoutput data, we estimate the technical coefficients to be $a_{c}=0.428, a_{s}=0.395$, and $a_{h}=0.335$, while the (net) import fraction $m=4.2 \%$. The other technical coefficients (which we ignore) are an order of magnitude smaller; the largest is expenditure by the hospital sector on goods and services of the social sector, with a technical coefficient of 0.028 .

The social sector only produces goods for consumption, $C_{s}$, adjusted by the sales tax rate $\tau_{s}^{\text {sales }}=6.0 \%$,

$$
X_{s}=\left(1-\tau_{s}^{\text {sales }}\right) C_{s}
$$

The consumption function for social goods and services is provided below.

Demand for hospital services, $C_{h}$, is, under normal circumstances, set as a fraction $h$ of the wage bill (set to $10 \%$, based on input-output data). During the pandemic, it is set to that rate multiplied by ratio of hospitalized patients to the normal level. The tax rate on hospital services is very low (about $0.2 \%$ ), and we ignore it. In terms of parameters introduced above, this gives

$$
X_{h}=h W\left[\frac{\left(1-\varepsilon^{\prime}\right) b N+\eta N_{i}}{(1-\varepsilon) b N}\right]
$$

This is the level of activity that drives intermediate demand for core goods. However, revenues are lower than this due to incomplete cost recovery for COVID-19 patients. We assume a marginal cost recovery rate for COVID-19 patients $r_{h}$, so that revenues $Y_{h}$ (and personal expenditure $C_{h}$ ) are equal to

$$
Y_{h}=C_{h}=h W \frac{1-\varepsilon^{\prime}}{1-\varepsilon}+r_{h}\left(X_{h}-h W \frac{1-\varepsilon^{\prime}}{1-\varepsilon}\right) .
$$

In model runs, we set $r_{h}=25 \%$.

Value added in each sector $V_{i}$ (at basic prices) is given by total revenue $Y_{i}$, less the cost of intermediate consumption,

${ }^{10}$ U.S. Bureau of Economic Analysis, "The Domestic Supply of Commodities by Industries" and "The Use of Commodities by Industries" https://www.bea.gov/industry/input-output-accounts-data (accessed 29 April 2020). We used the 15-industry table for 2018 , identifying the social sector as the sum of "Retail trade", "Arts, entertainment, recreation, accommodation, and food services", and "Other services, except government". We set "Hospital" as 36\% of "Educational services, health care, and social assistance" based on the breakdown in the 71-industry table. We net out the government sector (which largely buys and sells to and from itself), and separately track the government wage bill. 


$$
V_{i}=Y_{i}-a_{i} X_{i}
$$

For the core and social sectors, we assume full cost recovery, so $Y_{i}=X_{i}$ for those sectors. As discussed above, the hospital sector is not able to fully recover costs. Firms pay production taxes on their value added at a rate $\tau_{i}^{\text {prod }}$. Estimates from the national accounts give $\tau_{c}^{\text {prod }}=3.9 \%, \tau_{s}^{\text {prod }}=2.5 \%$, and $\tau_{h}^{\text {prod }}=1.9 \%$.

Firms are assumed to pay workers a fixed fraction $\omega_{i}$ of value added after paying for intermediate goods, with $\omega_{c}=48 \%, \omega_{s}=70 \%, \omega_{h}=81 \%$. For the hospital sector, we set wages based on revenue, so the wage bill in each sector is

$$
W_{c}=\omega_{c} V_{c}, \quad W_{s}=\omega_{s} V_{s}, \quad W_{h}=\omega_{h}\left(1-a_{h}\right) Y_{h}
$$

The sum of these gives the private wage bill $W_{\text {priv }}=W_{c}+W_{s}+W_{h}$. Added to this is the government wage bill $W_{g}$, which is a multiple $\omega_{g}$ of government expenditure on core goods, $W_{g}=\varpi_{g} G_{c}$, with $\varpi_{g}=1.24$. All wage income is assessed at a tax rate $\tau^{\text {wage }}$. We estimated the tax rate from the Bureau of Labor Statistics (BLS) Consumer Expenditure Survey (CEX) by taking total private tax payments plus the social security contribution divided by wages, salaries, and self-employment income. This gave a rate of $\tau^{\text {wage }}=27.7 \%$.

We allow for wages to be supplemented by unemployment benefits. We set baseline levels of the wage bill in each sector, $W_{i}^{\text {base }}$, which grow at the baseline rate $\gamma$. Unemployment is expected to be quite different in the different sectors, and the hospital sector may even see expanded employment. We therefore sum the sectoral wage gaps $\left(W_{i}^{\text {base }}-W_{i}\right)_{+}$. The notation $(x)_{+}$indicates the "positive part" function, equal to $x$ when it is positive and zero otherwise. Later, we will use the corresponding "negative part" function $(x)_{-}$, which is equal to $x$ when it is negative and zero otherwise. With this notation, we define unemployment benefits $U$ by

$$
U=\chi \sum_{i \in\{c, s, h\}}\left(W_{i}^{\text {base }}-W_{i}\right)_{+}
$$

The coefficient $\chi$ is the coverage of the wage gap. We assume different values for this parameter, below, to capture different stimulus strategies.

Profits $\Pi_{i}$ are the remainder from total output after wages, intermediate goods, and production taxes are paid for. If profits exceed investment, then it is distributed to households. Profits returned to households, $D$, whether directly as distributed profits or indirectly as repayment of loans, are given by the difference between profits $\Pi$ and investment $I$ plus the total of foreign, public, and net household saving (or, if the net is negative, zero). Foreign saving is equal to net imports $M=m\left[\left(1-\tau_{c}^{\text {sales }}\right) C_{c}+I\right]$. Because we include government expenditure on wages in the total wage bill, and calculate profits net of production taxes, the expression for net government saving relevant to this calculation is given by the sum of the wage tax $T_{w}$ and total sales taxes $T_{\text {sales}}$, net of government expenditure on core sector goods and services $G_{c}$. Net household saving, $S_{h}$, is the difference between income from all sources and consumption of goods and services. The result is

$$
D=\max \left(0, \Pi+M+S_{h}+T_{w}+T_{\text {sales }}-G_{c}-I\right) .
$$

\subsection{Consumption}

Personal consumption expenditure on hospital services is given by equation (24). For core and social goods and services, we take note of some peculiarities of the US. First, unlike other high-income countries, the US does not have universal health care. This is reflected in the input-output tables, where $100 \%$ of hospital expenditures are recorded under "Personal consumption expenditures". Second, total consumption 
expenditures exceeds the total wage bill (taken to equal "Compensation of employees", which includes non-wage compensation such as employer contributions to health insurance). So, although some households save out of wage income, we simplify the model design by assuming that all saving is out of profits. All wages are spent, as are distributed profits as given by equation (28).

We separate the consumption function in two parts. First, we define basic disposable income $Y_{d}$, which excludes profit income, as the sum of the wage bill $W$ and unemployment benefits $U$, net of hospital expenditures. A fraction $c_{0}$ of $Y_{d}$ is devoted to expenditures that are very difficult to reduce. These go beyond the list of shelter, food at home, utilities, health insurance, and medications, which as noted in the Introduction make up 39\% of expenditure, to include food away from home, home and car maintenance, cleaning supplies, loan payments, education, and clothing. Based on expenditure patterns in the US, we set this to $c_{0}=60 \%$ of $Y_{d}$. We set the initial value of these core expenditures to an irreducible minimum level $C_{c 0}=c_{0} Y_{d 0}$. Otherwise, basic core expenditures rise with basic disposable income and are equal to $c_{0} Y_{d}$.

Beyond the basic core consumption level, we assume that households spend on core goods at a marginal rate $c_{m}$ out of the remaining income and profits. Defining $c=c_{0}+\left(1-c_{0}\right) c_{m}$, we have

$$
C_{c}=\max \left\{C_{c 0}, c_{m} D+c\left[U+\left(1-\tau^{\text {wage }}\right) W-C_{h}\right]\right\} .
$$

We calibrated $c_{m}$ to get value added shares close to those reported in the input-output data, finding $c_{m}=60 \%$. This gives a value for $c$ of $80 \%$.

Expenditure on "social" goods depends on the fraction of the population that is infected or "visible", and on social distancing. We further assume (somewhat optimistically) that symptomatic individuals (proxied by visible cases) always avoid consuming social goods. Because many firms in the social sector can remain open to some degree (e.g., by adding delivery or take-out service, or by switching to an online format), we do not assume that social distancing translates one-to-one into a reduction in social sector expenditure. We introduce an economic impact quotient $q$. There is little basis for estimating this important parameter, which we set at $q=0.5$ in the model runs. Taking these factors into account gives an activity reduction factor $\varphi$, where

$$
\varphi=1-\max \left(q \sigma_{\mathrm{SD}}, \sigma_{\mathrm{IC}} \bar{f}_{i}, f_{v} \bar{f}_{i}\right)
$$

We then set consumption of social goods equal to

$$
C_{s}=\varphi \max \left\{0,\left(1-c_{m}\right) D+(1-c)\left[U+\left(1-\tau^{\text {wage }}\right) W-C_{h}\right]\right\} .
$$

This is the channel through which the COVID-19 epidemic impacts upon the economy. As social interaction is reduced or people become ill, they reduce expenditure on "social" goods and services. Through equation (21), this reduces demand for core goods and services as well. As economic activity declines, investment declines as well, as discussed below. This has a further, multiplier, effect on the economic activity. The result is a recession, underutilization of capital, and unemployed workers.

For any sector $i$, output is given by the capital stock multiplied by capital productivity and utilization,

$$
X_{i}=u_{i} \kappa_{i} K_{i}
$$


The capital stock in each sector is expanded through investment and depleted through depreciation, ${ }^{11}$

$$
\dot{K}_{i}=I_{i}-\delta K_{i} .
$$

We set the depreciation rate $\delta$ to 5\%/year, based on values from the Penn World Table 9.1 (Feenstra et al. 2015) and calibrated capital productivity to reproduce the GDP and investment expenditure reported in the input-output tables.

\subsection{Investment}

Total investment expenditure - and therefore the demand for investment goods - is the sum from all sectors,

$$
I=\sum_{i \in\{c, s, h\}} I_{i}
$$

Following standard post-Keynesian (neo-Kaleckian) theory (Kalecki 1969; Weintraub 1979; Dutt 1984; see Lavoie 2014), we assume an investment function that depends on capacity utilization (Lavoie 1995). The investment rate is above baseline when capacity is above its normal level and below baseline when it is below its normal level.

In the hospital sector, investment is expected to rise above baseline, which translates into increased bed capacity. We allow capacity utilization to rise well above $100 \%$ as hospitals stretch their available capacity to meet the crisis. However, they may be constrained by their supply chains: we limit capacity utilization in the core goods sector to $100 \%$. In the social sector, we expect capacity utilization to contract and investment to fall below baseline. In the core goods sector, utilization and investment can either rise or fall.

We assume that firms will, if they can, always replace depreciated capital. However, a certain number will not be able to sustain a long downturn, and will close down. In that case, they will have net negative investment, as their abandoned capital depreciates and is not replaced. We further assume that investment rates have a maximum limit. This applies to the hospital sector, which will face bottlenecks when trying to expand capacity if the number of cases rises rapidly, but which will be able to gradually expand capacity if the curve is flattened.

After the infection rate has passed its peak, firms start to build in an expectation that the economy will recover. This is reflected in a lower weight on utilization being below baseline. Investment is still depressed in that case, but to a lesser degree.

Taking all of these factors into account, the (gross) investment rate depends on utilization, profit rates, and common (net) long-run growth expectations in the following way,

$$
g_{i}^{i}=\frac{I_{i}}{K_{i}}=\delta+\min \left\{\theta \gamma, \max \left[-\varphi \delta, \gamma+\alpha\left(u_{i}-u_{d}\right)_{+}+\psi \alpha\left(u_{i}-u_{d}\right)_{-}\right]\right\} .
$$

Here, $u_{d}$ is a desired or target utilization level, which we set to 0.85 . The factor $\theta$ says how much larger investment can be over the normal rate, which is relevant to the hospital sector. There is no obvious source of data for this, and we set it equal to 1.1. The factor $\varphi$ is the fraction of firms that close down when utilization is low, set to $10 \%$, while the factor $\psi$ is equal to one before the infection peak and (optimistically) to zero afterwards. That is, after the peak, businesses act as though the economy will grow at its prior rate,

\footnotetext{
${ }^{11}$ In principle, these equations should be solved simultaneously. For simplicity, we use lagged values, but that means that we must anticipate one day's wage growth. We do that by using the lagged growth in the core sector capital stock.
} 
despite low consumer demand. We assume the sensitivity of the investment rate to the utilization rate, $\alpha$, is 0.25/year. This is similar to the total effect of sales on investment found by Fazzari and Mott (1986).

\subsection{Economic scenarios}

In addition to the macroeconomic baseline scenario, we ran three alternative scenarios. The first, Epi Macro II90 U10, ${ }^{12}$ features intensive testing and contact tracing to support a $90 \%$ effective isolation of infected individuals. Unemployment benefits cover 10\% of lost wages. The second, Epi Macro SD75 U10, is our best-fit epidemiological scenario as shown in Figure 1, in which contacts between susceptible and infective individuals are reduced by $75 \%$, with the additional assumption of $10 \%$ coverage of lost wages. The third, Epi Macro SD75 U100, assumes 100\% coverage of lost wages.

GDP relative to the baseline for the three epidemiological scenarios are shown in Figure 5. As seen in the figure, when infective individuals can be effectively isolated, the impact on the economy is minimal. When they cannot, social distancing has a substantial economic impact. With $10 \%$ of lost wages covered by unemployment, GDP falls by $5 \%$ relative to the baseline, close to the first-quarter decline in US GDP of $4.9 \%$. $^{13}$ According to the model developed in this paper, the economy falls no further than that, as consumption levels stabilize. However, the coincidence with observation should be treated cautiously: the precise value depends on uncertain parameters, in particular the economic impact quotient $q$. When $100 \%$ of wage loss is covered by unemployment benefits, the fall in GDP is one percentage point less, at $4 \%$.

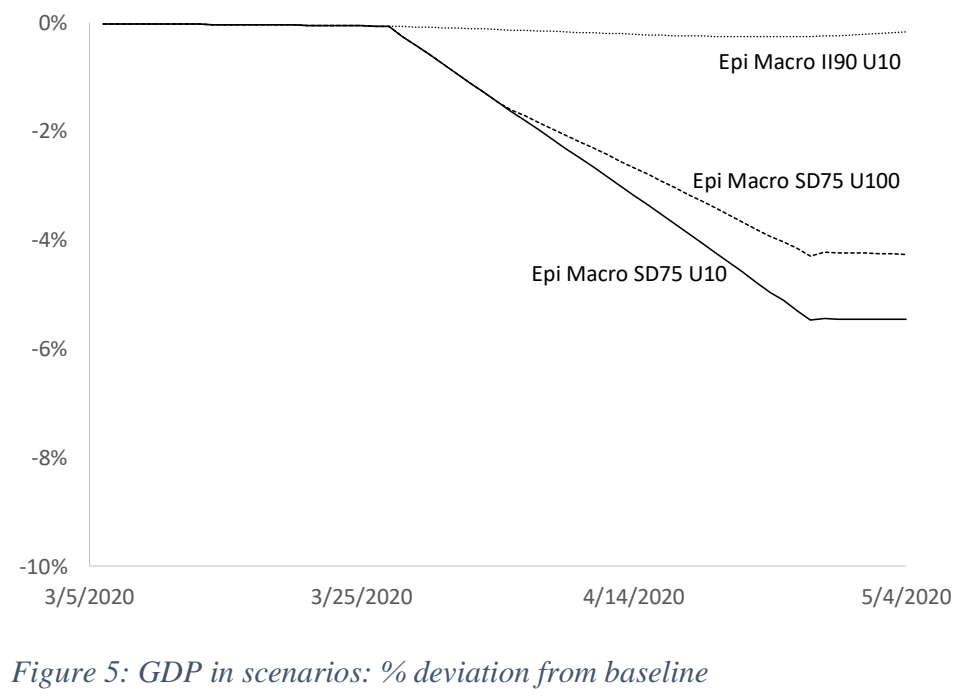

A few days after the contraction begins, the simulated average profit rate in the Epi Macro SD75 U10 scenario is $9.5 \%$ below baseline, due to falling profitability in the hospital sector and loss of income in the social sector. Over a period of several months (longer, but within a usual macroeconomic "short run"), the average profit rate is $5.2 \%$ below baseline, as COVID-19 cases recede and profits stabilize. The fall is less severe if $100 \%$ of lost wages is covered by unemployment: $7.8 \%$ in the short term and $2.7 \%$ over several months. Thus, covering unemployment helps support both wage-earners and those receiving profit income. Moreover, whether $10 \%$ or $100 \%$ of lost wages is covered by unemployment, cumulative household savings

\footnotetext{
${ }^{12}$ The difference between the "Epi" and "Epi Macro" scenarios is that bed capacity endogenously expands in the latter, while it remains fixed in the former. This has a small effect on mortality rates due to overflow.

${ }^{13}$ https://fred.stlouisfed.org/series/GDPC1, accessed 3 May 2020.
} 
in model simulations exceeds cumulative unemployment benefits. This suggests that the expansion of benefits can be funded by domestic savings.

There is much current discussion of the benefits of ending social distancing in order to mitigate economic impacts. Indeed, social distancing must end at some point. If it is replaced by a regime of testing and contact tracing that allows for effective separation of susceptible and infective individuals, then model simulations suggest that the economy can recover quickly. This is shown in Figure 6 in the scenario Epi Macro SD75 II90 U10, in which a 75\% effective social distancing regime (SD75) is followed by a $90 \%$ effective strategy of isolating infective cases (II90). The recovery occurs as people with employment or non-wage income expand their expenditure on social goods and services, allowing those workers to return to work. As can be seen in Figure 6, the recovery is unrealistically rapid in the simulation. In fact, a gradual opening up would be expected. Nevertheless, the simulations suggest that the economy can "bounce back".

If social distancing ends with no controls on the spread of the disease, then, as shown in Figure 4, deaths increase substantially. We do not show macroeconomic model runs for this scenario, because the model is not designed to capture the effects of an overwhelmed hospital system. Nevertheless, we report some of the broad indications. The large second wave of cases seen in Figure 4 is reflected, perversely, in a temporary boom - an expansion in GDP, after an initial dip. However, this is a misleading statistic. The boom is due to expansion of the core sector as it struggles to supply the overburdened hospital sector. In the simulations, profits, household saving, and demand for social sector goods and services collapse as households divert expenditure towards paying for hospital services.

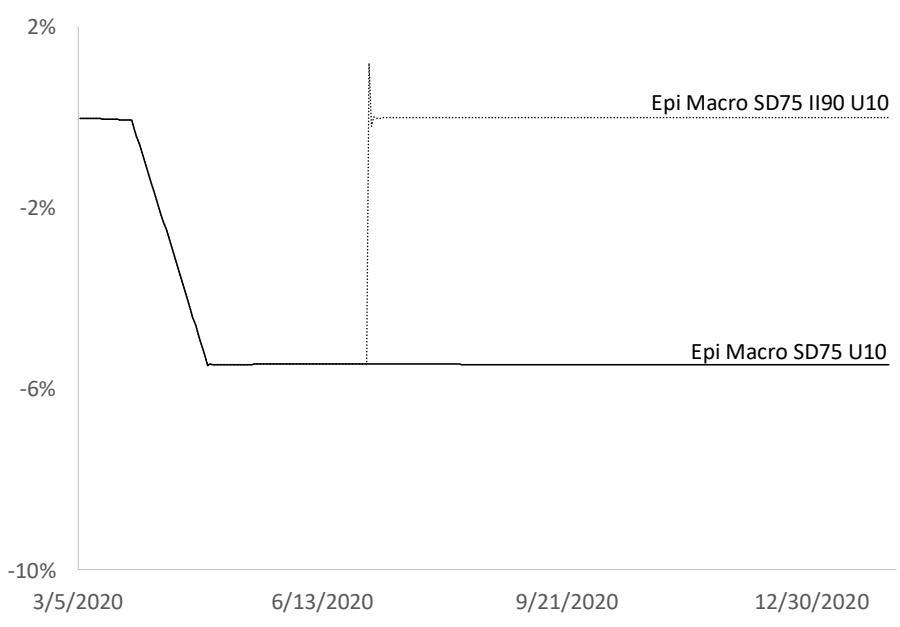

Figure 6: GDP: \% deviation from baseline under social distancing replaced with testing and tracing

\section{Discussion and conclusion}

In the US, political and policy debate on COVID-19 has centered on the economic impacts of public health measures. That debate can be informed by linked epidemiological-macroeconomic models. This paper presents such a model. Given the very high uncertainties surrounding the epidemic, the model is stylized and intended for broad scenario exploration rather than projection. The post-Keynesian model presented in this paper treats the impact of the pandemic as a "demand shock"; that is, as an externally-driven fall in demand for goods and services. Both aggregate supply and aggregate demand impacts are expected (Correia et al. 2020; Eichenbaum et al. 2020), but in contrast to some recent neoclassical treatments (Fornaro and Wolf 2020; Guerrieri et al. 2020), we view the epidemic as primarily affecting demand. 
Several scenarios are presented. One that features aggressive testing and contact tracing has minimal impacts on the economy while keeping deaths extremely low. This finding underscores the consistent message from public health experts that testing and tracing are preconditions for safely reopening the economy. Without it, cases and deaths can rise rapidly when the economy reopens as the large remaining susceptible population encounters the small but persistent infective population. ${ }^{14}$ This severely burdens the hospital system. In a scenario with no testing or contact tracing, but with strong social distancing, the model economy experiences a sharp and deep recession.

The fall in GDP arises in the model through a Keynesian mechanism. Demand falls for goods and services provided by "social" businesses such as restaurants, due to social distancing or illness, while consumer demand for "core" goods and services is crowded out by rising hospital costs. This decline in primary demand leads to secondary impacts as wages fall, demand for intermediate goods contracts, and investment slows. The initial drop is thus amplified through wage, intermediate goods, and investment multipliers.

The Keynesian remedy is fiscal stimulus, while the epidemiological remedy is testing and contact tracing (at least until a vaccine is developed). The model runs suggest that the current combination of social distancing and fiscal stimulus, in the form of wage support, are having the desired effect of constraining mortality and mitigating economic harm. In combination, fiscal stimulus and testing are very effective. Fiscal stimulus keeps households out of debt and mitigates the recession until the testing and tracing regime is in place. Without testing and tracing there is a very large number of deaths. Without fiscal stimulus, households who have lost income due to public health measures will be unable to make needed expenditures on food, rent, medications and other essentials.

The sustainability of deficit spending is a contentious topic. A prominent strand of post-Keynesian theory, Modern Monetary Theory, argues that constraints on central government expenditure are weak to nonexistent (Wray 1998). The theory is controversial even among post-Keynesians (Fullbrook and Morgan 2019; Juniper et al. 2014; Lavoie 2019). Yet, there is no disagreement that deficit spending is an effective way to stimulate a depressed economy. Moreover, the model runs suggest that excess saving due to curtailed expenditure by the still-employed population is more than enough to fund the wages lost by the unemployed population. The federal government must take on additional debt, but the debt will be owed to its citizens. The simulations also show that, by sustaining expenditure, unemployment coverage mitigates the loss of profits.

The simulation results are reassuring, but only if a thorough testing and contact tracing regime is put in place. If it is, then wage supports are needed in the interim, but they can be rapidly scaled back once infective individuals can be identified and isolated. In that case, social distancing measures can be relaxed and the model economy recovers rapidly. However, if the economy is reopened without the ability to identify and isolate infective cases through testing and contact tracing, then the number of deaths can rise rapidly, with severe economic impacts and a disastrous human toll.

\footnotetext{
${ }^{14}$ In the public debate, two camps have been identified: "big-denominator" and "the cases are the cases". The first proposes that large numbers of people have already been exposed but were asymptomatic. Adherents suggest that because we may have a large invisible pool of recovered cases, we may be close to the point of herd immunity and so should open up. The "cases are the cases" viewpoint is that the only data we have is the documented cases and it would be irresponsible to act on supposition. In the model in this paper, we allow for a substantial number of asymptomatic individuals ( $87 \%$ of infective cases), but they are carriers of the disease. The model in this paper thus does not go as far as "the cases are the cases", but nevertheless the results do not support the big-denominator hypothesis.
} 


\section{References}

Baud, D., Qi, X., Nielsen-Saines, K., Musso, D., Pomar, L. and Favre, G. (2020). Real estimates of mortality following COVID-19 infection. The Lancet. Infectious Diseases. DOI: 10.1016/S1473-3099(20)30195-X

Chatterjee, K., Chatterjee, K., Kumar, A. and Shankar, S. (2020). Healthcare impact of COVID-19 epidemic in India: A stochastic mathematical model. Medical Journal Armed Forces India. DOI: 10.1016/j.mjafi.2020.03.022

Chowell, G., Chowell, D., Roosa, K., Dhillon, R. and Srikrishna, D. (2020). Sustainable social distancing through facemask use and testing during the Covid-19 pandemic. MedRxiv. 2020.04.01.20049981. DOI:

$10.1101 / 2020.04 .01 .20049981$

Correia, S., Luck, S. and Verner, E. (2020). Pandemics Depress the Economy, Public Health Interventions Do Not: Evidence from the 1918 Flu. ID 3561560. Social Science Research Network, Rochester, NY. DOI: $10.2139 / \mathrm{ssrn} .3561560$

Dutt, A. K. (1984). Stagnation, income distribution and monopoly power. Cambridge Journal of Economics, 8(1). 25-40.

Eichenbaum, M. S., Rebelo, S. and Trabandt, M. (2020). The Macroeconomics of Epidemics. Working Paper Series, 26882. National Bureau of Economic Research. DOI: 10.3386/w26882

Fang, Y., Nie, Y. and Penny, M. (2020). Transmission dynamics of the COVID-19 outbreak and effectiveness of government interventions: A data-driven analysis. Journal of Medical Virology, n/a(n/a). DOI: 10.1002/jmv.25750

Fazzari, S. M. and Mott, T. L. (1986). The investment theories of Kalecki and Keynes: an empirical study of firm data, 1970-1982. Journal of Post Keynesian Economics, 9(2). 171-187. http://www.tandfonline.com/doi/pdf/10.1080/01603477.1986.11489611

Feenstra, R. C., Inklaar, R. and Timmer, M. P. (2015). The next generation of the Penn World Table. American Economic Review, 105(10). 3150-82. DOI: 10.1257/aer.20130954

Fornaro, L. and Wolf, M. (2020). Covid-19 Coronavirus and Macroeconomic Policy. Barcelona GSE Working Paper Series, 1168. Barcelona Graduate School of Economics, Barcelona, Spain

Fullbrook, E. and Morgan, J. (2019). Introduction: whither MMT? Real-World Economics Review, 89. 2-4.

Grant, A. (2020). Dynamics of COVID-19 epidemics: SEIR models underestimate peak infection rates and overestimate epidemic duration. MedRxiv. 2020.04.02.20050674. DOI: 10.1101/2020.04.02.20050674

Guerrieri, V., Lorenzoni, G., Straub, L. and Werning, I. (2020). Macroeconomic Implications of COVID-19: Can Negative Supply Shocks Cause Demand Shortages? Working Paper Series, 26918. National Bureau of Economic Research. DOI: 10.3386/w26918

Gupta, R., Pandey, G., Chaudhary, P. and Pal, S. K. (2020). SEIR and Regression Model based COVID-19 outbreak predictions in India. MedRxiv. 2020.04.01.20049825. DOI: 10.1101/2020.04.01.20049825

IHME COVID-19 health service utilization forecasting Team and Murray, C. J. (2020). Forecasting COVID-19 impact on hospital bed-days, ICU-days, ventilator-days and deaths by US state in the next 4 months. MedRxiv. 2020.03.27.20043752. DOI: 10.1101/2020.03.27.20043752

Janz, N. K. and Becker, M. H. (1984). The Health Belief Model: a decade later. Health Education Quarterly, 11(1). 1-47. DOI: $10.1177 / 109019818401100101$

Ji, Y., Ma, Z., Peppelenbosch, M. P. and Pan, Q. (2020). Potential association between COVID-19 mortality and health-care resource availability. The Lancet Global Health, 8(4). e480. DOI: 10.1016/S2214-109X(20)30068-1 
Juniper, J., Sharpe, T. P. and Watts, M. J. (2014). Modern monetary theory: contributions and critics. Journal of Post Keynesian Economics, 37(2). 281-307. DOI: 10.2753/PKE0160-3477370205.2015.11082991

Kalecki, M. (1969). Theory of Economic Dynamics: An Essay on Cyclical and Lon-Run Changes in Capitalist Economy. Augustus M. Kelly, New York, NY, US

Kermack, W. O. and McKendrick, A. G. (1927). A contribution to the mathematical theory of epidemics. Proceedings of the Royal Society of London. Series A, Containing Papers of a Mathematical and Physical Character, 115(772). 700-721. https://www.jstor.org/stable/94815

King, J. E. (2012). The Microfoundations Delusion: Metaphor and Dogma in the History of Macroeconomics. Edward Elgar Pub, Cheltenham, UK

Kirman, A. (2014). Is it rational to have rational expectations? Mind \& Society, 13(1). 29-48. DOI: 10.1007/s11299014-0136-x

Lauer, S. A., Grantz, K. H., Bi, Q., Jones, F. K., Zheng, Q., Meredith, H. R., Azman, A. S., Reich, N. G. and Lessler, J. (2020). The incubation period of coronavirus disease 2019 (COVID-19) from publicly reported confirmed cases: estimation and application. Annals of Internal Medicine. DOI: 10.7326/M20-0504

Lavoie, M. (1995). The Kaleckian model of growth and distribution and its neo-Ricardian and neo-Marxian critiques. Cambridge Journal of Economics, 19(6). 789-818.

Lavoie, M. (2014). Post-Keynesian Economics: New Foundations. Edward Elgar Publishing Limited, Cheltenham UK

Lavoie, M. (2019). Modern monetary theory and post-Keynesian economics. Real-World Economics Review, 89. 97-108.

Li, Q. and Feng, W. (2020). Trend and forecasting of the COVID-19 outbreak in China. DOI:

10.1016/j.jinf.2020.02.014

Liu, Z. and Guo, W. (2020). Government responses matter: predicting Covid-19 cases in US under an empirical Bayesian time series framework. MedRxiv. 2020.03.28.20044578. DOI: 10.1101/2020.03.28.20044578

Lloyd, A. L. (2001a). Destabilization of epidemic models with the inclusion of realistic distributions of infectious periods. Proceedings of the Royal Society B: Biological Sciences, 268(1470). 985-93. DOI: 10.1098/rspb.2001.1599

Lloyd, A. L. (2001b). Realistic distributions of infectious periods in epidemic models: changing patterns of persistence and dynamics. Theoretical Population Biology, 60(1). 59-71. DOI: 10.1006/tpbi.2001.1525

Matrajt, L. and Leung, T. (2020). Evaluating the effectiveness of social distancing interventions against COVID-19. MedRxiv. 2020.03.27.20044891. DOI: 10.1101/2020.03.27.20044891

Menendez, J. (2020). Elementary time-delay dynamics of COVID-19 disease. MedRxiv. 2020.03.27.20045328. DOI: $10.1101 / 2020.03 .27 .20045328$

Moghadas, S. M., Shoukat, A., Fitzpatrick, M. C., Wells, C. R., Sah, P., et al. (2020). Projecting hospital utilization during the COVID-19 outbreaks in the United States. Proceedings of the National Academy of Sciences, 117(16). 9122-26. DOI: 10.1073/pnas.2004064117

Morin, B. R., Kinzig, A. P., Levin, S. A. and Perrings, C. A. (2018). Economic incentives in the socially optimal management of infectious disease: when R0 is not enough. EcoHealth, 15(2). 274-89. DOI: 10.1007/s10393-017$1270-9$ 
Rocklöv, J., Sjödin, H. and Wilder-Smith, A. (2020). COVID-19 outbreak on the Diamond Princess cruise ship : estimating the epidemic potential and effectiveness of public health countermeasures. Journal of Travel Medicine. http://urn.kb.se/resolve?urn=urn:nbn:se:umu:diva- 168517

Shaikh, A. (2016). Capitalism: Competition, Conflict, Crises. Oxford University Press, New York, NY, US

Solow, R. (2008). The state of macroeconomics. The Journal of Economic Perspectives, 22(1). 243-46. http://www.jstor.org/stable/27648233

Summers, L. H. (2014). U.S. economic prospects: secular stagnation, hysteresis, and the zero lower bound. Business Economics, 49(2). 65-73. DOI: 10.1057/be.2014.13

Teulings, C. and Baldwin, R., eds. (2014). Secular Stagnation: Facts, Causes and Cures. Centre for Economic Policy Research, London, UK. http://www.tau.ac.il/ yashiv/Vox_secular_stagnation.pdf

Wang, W., Tang, J. and Wei, F. (2020). Updated understanding of the outbreak of 2019 novel coronavirus (2019$\mathrm{nCoV}$ ) in Wuhan, China. Journal of Medical Virology, 92(4). 441-47. DOI: 10.1002/jmv.25689

Wearing, H. J., Rohani, P. and Keeling, M. J. (2005). Appropriate models for the management of infectious diseases. PLoS Medicine, 2(7). e174. DOI: 10.1371/journal.pmed.0020174

Weintraub, S. (1979). Generalizing Kalecki and simplifying macroeconomics. Journal of Post Keynesian Economics, 1(3). 101-6. http://www.jstor.org/stable/4537486

Wray, L. R. (1998). Understanding Modern Money: The Key to Full Employment and Price Stability. Edward Elgar, Cheltenham, Glos, UK ; Northampton, MA

Zhan, C., Tse, C. K., Lai, Z., Chen, X. and Mo, M. (2020). General model for COVID-19 spreading with consideration of intercity migration, insufficient testing and active intervention: application to study of pandemic progression in Japan and USA. MedRxiv. 2020.03.25.20043380. DOI: 10.1101/2020.03.25.20043380

Zhang, G., Pang, H., Xue, Y., Zhou, Y. and Wang, R. (2020). Forecasting and Analysis of Time Variation of Parameters of COVID-19 Infection in China Using an Improved SEIR Model. In Review. DOI: 10.21203/rs.3.rs$16159 / \mathrm{v} 1$ 